Pacific Journal of Mathematics

PROVABLE EQUALITY IN PRIMITIVE RECURSIVE
ARITHMETIC WITH AND WITHOUT INDUCTION 


\section{PROVABLE EQUALITY IN PRIMITIVE RECURSIVE ARITHMETIC WITH AND WITHOUT INDUCTION}

\section{HARVEY FRIEDMAN}

The set of identities provable in primitive recursive arithmetic without induction (PRE), with or without standard quantifier free successor axioms, is recursive. A finite number of identities can be added to $\mathrm{PRE}$ such that the set of identities provable become complete $r$.e. (with or without successor axioms). If the successor axiom $y \neq 0 \rightarrow \exists x(S(x)=y)$ is added to $\mathrm{PRE}$, then the set of identities provable become complete r.e. (with or without $1 \neq 0$ ). If $\mathrm{PRE}$ is augmented by definition by cases, then the set of identities provable become complete r.e. (with or without successor axioms). Equivalents of primitive recursive arithmetic (PRA) are given, involving the rule of induction restricted to identities.

We begin with the definition of the $n$-ary primitive recursion indices, $0<n$. These are given by (i) 0,1 are 1 -ary p.r. indices. (ii) $(2, n, m), 1 \leqq m \leqq n$, is an $n$-ary p.r. index. (iii) $\left(3, x, y_{1}, \cdots, y_{k}\right)$ is an $n$-ary p.r. index if $y_{1}, \cdots, y_{k}$ are $n$-ary p.r. indices and $x$ is a $k$-ary p.r. index, $1 \leqq n, k$. (iv) $(4, x, y)$ is a 1 -ary p.r. index if $y$ is a 2-ary p.r. index. (v) $(5, x, y)$ is an $n$-ary p.r. index if $x$ is an $(n-1)$-ary p.r. index and $y$ is an $n+1$-ary p.r. index, $1<n$.

The $n$-ary primitive recursive function symbols are the expressions $F_{x}$, where $x$ is an $n$-ary p.r. index. We let $S$ abbreviate $F_{1}$, and $\overline{0}$ be $0, \overline{n+1}$ be $S(\bar{n})$.

The theory PRE (primitive recursion equations) is given by $F_{0}(a)=0, F_{(2, n, m)}\left(a_{1}, \cdots, a_{n}\right)=a_{m}, F_{\left(3, x, y_{1}, \cdots y_{k}\right)}\left(a_{1}, \cdots, a_{n}\right)=F_{x}\left(F_{y_{1}}\left(a_{1}\right.\right.$, $\left.\left.\cdots, a_{n}\right), \cdots, F_{y_{k}}\left(a_{1}, \cdots, a_{n}\right)\right), F_{(4, x, y)}(0)=\bar{x}, F_{(4, x, y)}(S(a))=F_{y}\left(a, F_{(4, x, y)}(a)\right)$, $F_{(5, z, w)}\left(a_{1}, \cdots, a_{n}, 0\right)=F_{z}\left(a_{1}, \cdots, a_{n}\right), F_{(5, z, w)}\left(a_{1}, \cdots, a_{n}, S\left(a_{n+1}\right)\right)=F_{w}\left(a_{1}\right.$, $\left.\cdots, a_{n}, a_{n+1}, F_{(5, z, w)}\left(a_{1}, \cdots, a_{n}, a_{n+1}\right)\right)$, where $(2, n, m),\left(3, x, y_{1}, \cdots, y_{k}\right)$, $(4, x, y)$, and $(5, z, w)$ are respectively $n$-ary, $n$-ary, 1 -ary, and $n+1$ ary p.r. indices, $1 \leqq n$.

We will firstly consider relationships between various successor axioms.

LEMMA 1. PRE $\vdash S(x)=S(y) \rightarrow x=y . \quad$ PRE $\vdash S(x)=0 \rightarrow y=0$.

Proof. Let $P$ be such that PRE $\vdash(P(0)=0 \quad \& \quad P(S(x))=x)$. Then PRE $\vdash(S(x)=S(y) \rightarrow P(S(x))=P(S(y)) \rightarrow x=y)$. Let $F$ be such that PRE $\vdash(H(y, 0)=0 \& H(y, S(x))=y)$. Then PRE $\vdash(S(x)=$ $0 \rightarrow F(y, 0)=y \rightarrow y=0)$. 
TEOREM 1. PRE $\vdash(S(x)=S(y) \rightarrow x=y)$. In addition, the following are provably equivalent in PRE. (i) $(\exists x)(x \neq 0)$. (ii) $(\exists x)(S(x) \neq x)$. (iii) $(\exists x)(S(x) \neq 0)$. (iv) $S(0) \neq 0 . \quad$ (v) $S(x) \neq 0$. $\quad \mathrm{PRE}+S(0) \neq 0+$ $(y \neq 0 \rightarrow(\exists x)(S(x)=y)) \vdash S(x) \neq x$. PRE $+S(0) \neq 0$ does not prove $S(x) \neq x$. PRE $+S(x) \neq x$ does not prove $(y \neq 0 \rightarrow(\exists x)(S(x)=y))$. $\mathrm{PRE}+(y \neq 0 \rightarrow(\exists x)(S(x)=y))$ does not prove $S(0) \neq 0$. PRE $+(y \neq$ $0 \rightarrow(\exists x)(S(x)=y))$ has a quantifier free axiomatization.

Proof. The first part is Lemma 1. For the second part, note that (v) $\rightarrow$ (iv) $\rightarrow$ (iii) $\rightarrow$ (i). By Lemma 1, (i) $\rightarrow$ (v). Thus (i), (iii), (iv), (v) are equivalent. Clearly (iv) $\rightarrow$ (ii). To see that (ii) $\rightarrow$ (iv), let PRE $\vdash(x+0=x \& x+S(y)=S(x+y))$. Then PRE $\vdash(S(0)=0 \rightarrow$ $x+S(0)=x+0=x=S(x+0)=S(x))$.

We now argue in PRE $+S(0) \neq 0+(y \neq 0 \rightarrow(\exists x)(S(x)=y))$. Let $F(0)=S(0), F(S(x))=0$. Then $y \neq 0 \rightarrow F(y)=0$. Hence $F(y) \neq y$. Let $E(0)=0, E(S(x))=F(E(x))$. Then $S(x)=x \rightarrow E(x)=F(E(x))$. But $F(E(x)) \neq E(x)$. So $S(x) \neq x$.

We sketch a proof that PRE $+S(0) \neq 0$ does not prove $S(x) \neq x$. Consider the type free $\lambda$-calculus with the three constant $0, S, R$, whose axioms and rules are given by the same clauses as those for the $\lambda$-calculus of [1], except that we add the axioms $R \alpha \beta 0=\beta$, $R \alpha \beta(S \gamma)=\alpha \gamma(R \alpha \beta \gamma)$, for all terms $\alpha, \beta, \gamma$. Then for cach p.r. function symbol $F$, we may naturally associate a closed term $F^{*}$ in such a way that the set of equivalence classes $[\alpha]$ of closed terms under convertibility form a model of PRE, where 0 is interpreted as [0], and $F\left(\left[\alpha_{1}\right], \cdots,\left[\alpha_{k}\right]\right)$ as $\left[F^{*} \alpha_{1} \cdots \alpha_{k}\right]$. This model will obey $(\exists x)(S x=x)$ by the fixed point theorem. It is also clear that $S(0) \neq 0$ also holds, since $S(0)$ is not convertible to 0 (by the Church Rosser theorem).

It will be established in Theorem 2 that $\mathrm{PRE}+S(x) \neq x$ does not prove $(y \neq 0 \rightarrow(\exists x)(S(x)=y))$.

The last independence result is obvious, using the one element model of PRE.

$\mathrm{PRE}+(y \neq 0 \rightarrow(\exists x)(S(x)=y))$ has the axiomatization PRE + $(y=0 \vee S(P(y))=y)$, where $P$ is as in the proof of Lemma 1 (see Lemma 11).

A set $X$ of p.r. function symbols is said to be closed just in case (1) $S \in X$. (2) if $F_{\left(3, x, y_{1}, \cdots, y_{k}\right)} \in X$ then $F_{x}, F_{y_{1}}, \cdots, F_{y_{k}} \in X$. if $F_{(4, x, y)} \in X$ then $F_{y} \in X$. (4) if $F_{(5, x, y)} \in X$ then $F_{x}, F_{y} \in X$. Let PRE be the set of all p.r. function symbols.

For closed $X$, we let $\mathrm{PRE}_{X}$ consist of those axioms of PRE all of whose function symbols are from $X$. An $X$-term is a term in the language of PRE, all of whose function symbols are from $X$. Below, $\alpha, \beta$ will denote terms in the language of PRE. 
We say that $\alpha$ is the atomic reduct of $\beta$ just in case one of the following holds: (a) $\alpha$ is $F_{0}(\gamma)$ and $\beta$ is 0 . (b) $\alpha$ is $F_{(2, n, m i}\left(\alpha_{1}, \cdots, \alpha_{n}\right)$ and $\beta$ is $\alpha_{m}$. (c) $\alpha$ is $F_{\left(3, x, y_{1}, \cdots, y_{k}\right)}\left(\alpha_{1}, \cdots, \alpha_{n}\right)$ and $\beta$ is

$$
F_{x}\left(F_{y_{1}}\left(\alpha_{1}, \cdots, \alpha_{n}\right), \cdots, F_{y_{k}}\left(\alpha_{1}, \cdots, \alpha_{n}\right)\right) \text {. }
$$

(d) $\alpha$ is $F_{(4, x, y)}(0)$ and $\beta$ is $\bar{x}$. (e) $\alpha$ is $F_{(5, x, y)}\left(\alpha_{1}, \cdots, \alpha_{n}, 0\right)$ and $\beta$ is $F_{x}\left(\alpha_{1}, \cdots, \alpha_{n}\right)$. (f) $\alpha$ is $F_{(5, x, y)}\left(\alpha_{1}, \cdots, \alpha_{n}, S\left(\alpha_{n+1}\right)\right)$ and $\beta$ is

$$
F_{y}\left(\alpha_{1}, \cdots, \alpha_{n}, \alpha_{n+1}, F_{(5, x, y)}\left(a_{1}, \cdots, \alpha_{n}, \alpha_{n+1}\right)\right) .
$$

We say that $\alpha$ is an immediate reduct of $\beta$ just in case $\alpha$ is the result of replacing one particular occurrence of a subterm $\gamma$ of $\beta$ by the atomic reduct of $\gamma$.

A sequence of terms $\left\{\alpha_{n}\right\}$ is called a reduction sequence just in case each $\alpha_{n+1}$ is an immediate reduct of $\alpha_{n}$. Observe that if $\alpha_{0}$ is an $X$-term, then each $\alpha_{n}$ must be an $X$-term.

Lemma 2. Every reduction sequence is finite in length. Any two maximal reduction sequences starting with $\alpha$, end in the same term.

Proof. This can either be obtained as a consequence of [4], or proved directly by the same methods. Also see [6].

Let us call $\alpha$ a reduct of $\beta$ just in case $\alpha$ is a term in some reduction sequence beginning with $\beta$. If $\gamma$ has no immediate reduct, then $\gamma$ is called irreducible.

The following is immediate from Lemma 2.

Lemma 3. To each PR-term $\alpha$, there is a unique irreducible term $\alpha^{*}$ which is a reduct of $\alpha$. Moreover, $\alpha^{*}$ can be effectively obtained from $\alpha$. If $\alpha$ is an X-term, $X$ closed, then $\alpha^{*}$ is an X-tem.

Lemma 4. Let $X$ be closed. If $\alpha^{*}=\beta^{*}$, and $\alpha, \beta$ are $X$-terms, then $\mathrm{PRE}_{X} \vdash \alpha=\beta$.

Proof. Show by induction on $n$, that if $\left(\alpha=\alpha_{0}, \alpha_{1}, \cdots, \alpha_{n}\right)$ is a reduction sequence, then $\mathrm{PRE}_{X} \vdash \alpha=\alpha_{n}$.

Fix $X$ to be a closed set of p.r. function symbols. We define the structure $\mathscr{A}_{X}$ so that (a) the domain is the set of all irreducible $X$-terms. (b) each $n$-ary $F \in X$ is interpreted by: $F\left(\alpha_{1}, \cdots, \alpha_{n}\right)$ is the term $\left(F\left(\alpha_{1}, \cdots, \alpha_{n}\right)\right)^{*}$. (c) $\mathscr{A}_{X}$ interprets the constant 0 as the term 0 , and interprets no other symbols. It is easy to see that $\mathscr{A}_{x} \vDash \mathrm{PRE}_{x}$.

Lemma 5. $\mathscr{A}_{X}$ interprets an irreducible X-term $\alpha$ as $\alpha$, under 
the valuations which interpretes each variable $x$ as the term $x$.

Proof. Use induction on the construction of the irreducible term $\alpha$, and the fact that subterms of irreducible terms are irreducible.

Lemma 6. For $X$-terms $\alpha, \beta$, if $\mathscr{A}_{X} \vDash \alpha=\beta$ then $\alpha^{*}=\beta^{*}$.

Proof. Suppose $\mathscr{A}_{X} \vDash \alpha=\beta$. Then $\mathscr{A}_{X} \vDash \alpha^{*}=\beta^{*}$, by Lemma 4. Hence $\alpha^{*}=\beta^{*}$, by Lemma 5 .

THEOREM 2. Let $X$ be a closed set of p.r. function symbols. For $X$-terms $\alpha, \beta$, the following are equivalent. (i) $\alpha^{*}=\beta^{*}$. (ii) $\mathrm{PRE}_{X} \vdash$ $\alpha=\beta$. (iii) $\operatorname{PRE} \cup\{S(x) \neq x,(\exists x)(x \neq 0 \&(\forall y)(S(y) \neq x))\} \vdash \alpha=\beta$.

Proof. (i) $\rightarrow$ (ii) is by Lemma 3 (ii) $\rightarrow$ (iii) is obvious. To see that (iii) $\rightarrow$ (i), it suffices to quote Lemma 6 , and show that $\mathscr{A}_{X} \vDash$ $(S(x) \neq x \&(\exists x)(x \neq 0 \&(\forall y)(S(y) \neq x)))$. The latter is a consequence of the fact that $S(\alpha)$ is always irreducible if $\alpha$ is.

Corollary 1. Let $X$ be a closed set of p.r. function symbols. Then PRE and $\mathrm{PRE}_{X}$ have the same set of consequences of the form $\alpha=\beta$, for $X$-terms $\alpha, \beta$, and this set is recursive.

The rest of this paper is devoted to undecidability results. We first present two recursion theoretic lemmas. Recall the definition of effectively inseparable sets in [3].

LEMMA 7. There are effectively inseparable recursively enumerable sets $A, B \subset \omega$. If $A, B$ are effectively inseparable recursively enumerable sets, $A \subset C, C \cap B=\varnothing$, and $C$ is recursively enumerable, then $C$ is creative.

Proof. The existence of $A, B$ is proved in [3]. The rest is immediate.

We fix $A, B$.

LEMMA 8. Every nonempty r.e. set is the range of some unary primitive recursive function. There is a primitive recursive binary function $g(n, m)$ such that $n \in B \leftrightarrow(\exists m)(g(n, m)=1)$, and $g$ is 0, 1valued.

Proof. Use the Kleene T-predicate.

Recally that a theory $T$ is called essentially undecidable just 
in case it is consistent, and any extension of $T$ whose set of consequences in the language of $T$ is recursive, is inconsistent. We will call a theory $T$ equationally essentially undecidable just in case it does not imply $x=y$, and any extension of $T$ whose set of equational consequences in the language of $T$ is recursive implies $x=y$.

To our knowledge, the existence of finitely axiomatized equationally essentially undecidable theories was first proved in [2], where it is shown that $C L$, a system of combinatory logic, viewed as an equational theory, is equationally essentially undecidable. Also, see [1].

In fact, one can see that $C L$ has an even stronger property: let $T$ be an extension of $C L$ not implying $x=y$. If the set of consequences of $T$ in the language of $C L$ is r.e., the set is complete r.e.. We call such a theory completely equationally essentially undecidable. ${ }^{1}$

An $n$-ary p.r. function symbol $F$ is said to represent the $n$-ary function $f$ just in case $f\left(x_{1}, \cdots, x_{n}\right)=y$ implies $\operatorname{PRE} \vdash F\left(\bar{x}_{1}, \cdots, \bar{x}_{n}\right)=\bar{y}$.

LEMMA 9. If $f$ is an n-ary primitive recursive function, then $f$ is represented by some p.r. function symbol $F$.

Proof. This is well known.

By Lemma 8 fix $f$ to be a unary primitive recursive function whose range is $A$. By Lemma 9 , fix $F, G$ to be p.r. function symbols which respectively represent $f, g$ (of Lemma 8 ).

A glance at the proof of the second part of Lemma 1 reveals that there is a function symbol $H$ such that for the closed $X$ with $H \in X, \mathrm{PRE}_{X} \vdash S(x)=0 \rightarrow y=0$.

LEMMA 10. Let $X$ be a closed set of p.r. function symbols, $F, G, H \in X . \quad$ If $n \in A$ then $\mathrm{PRE}_{X}+G(F(x), y)=0 \vdash G(\bar{n}, y)=0 . \quad$ If $n \in B$ then $\mathrm{PRE}_{X}+G(F(x), y)=0 \vdash(\exists y)(G(\bar{n}, y)=S(0))$.

Proof. If $n \in A$ then $n \in \mathrm{Rng}(f)$. Let $f(k)=n$. Then $\mathrm{PRE}_{X} \vdash$ $F(\bar{k})=\bar{n}$. Hence PRE $\mathrm{PR}_{X}+G(F(x), y)=0 \vdash G(\bar{n}, y)=0$. If $n \in B$ then $(\exists m)(g(n, m)=1)$. Hence $\operatorname{PRE}_{X} \vdash G(\bar{n}, \bar{m})=S(0)$. Hence

$$
\mathrm{PRE}_{X} \vdash(\exists y)(G(\bar{n}, y)=S(0)) \text {. }
$$

1 There are no essentially undecidable equational theories, since every consistent equational theory has a unique model with one element. If $T$ is equationally essentially undecidable, then $T+\exists x \exists y(x \neq y)$ is essentially undecidable. If $T$ is undecidable in either of the three senses, and $T$ extends $T^{\prime}$, then $T^{\prime}$ is undecidable in that same sense (even if the language of $T^{\prime}$ extends the language of $T$ ). If the same theory $T$ is viewed as having different languages, then these three notions of undeciability remain unaffected (as long as the languages in question are recursive). 
THOREM 3. PRE can be extended by a finite number of equations in its language, so that the result is a completely equationally essentially undecidable theory. Moreover, the same is true of any $\mathrm{PRE}_{X}$, provided $X$ is a closed set of p.r. function symbols containing a fixed finite list of p.r. function symbols.

Proof. Let $X$ be closed, $F, G, H \in X$. Let $T$ be an extension of $\mathrm{PRE}_{X}+G(F(x), y)=0$ not implying $x=y$. Let

$$
C=\{n: T \vdash G(\bar{n}, y)=0\} .
$$

By Lemma 10, $A \subset C$. Let $n \in B$. By Lemma 10, $T \vdash(\exists y)(G(\bar{n}, y)=S(0))$. If $n \in C$ then $T \vdash S(0)=0$, and hence $T \vdash x=y$. Hence $n \notin C$. So $B \cap C=\varnothing$. Assume that the set of equational consequences of $T$ in the language of $\mathrm{PRE}_{X}$ is r.e. Then $C$ is r.e. By Lemma 7, $C$ is complete r.e. Hence the set of equational consequences of $T$ in the language of $\mathrm{PRE}_{X}$ is complete r.e. To see that $\mathrm{PRE}_{X}+G(F(x), y)=0$ does not imply $x=y$, note that is has a (unique) model with domain $\omega$, in which 0 is interpreted as 0 and $S$ is interpreted as successor.

COROLlaRY 2. There is a finitely axiomatized equational theory which is completely equationally essentially undecidable, and which has a model with domain $\omega$ in which all function symbols are interpreted (primitive) recursively.

Now let $i, j$ be binary primitive recursive functions such that $i(n, 0)=1, A=\{n:(\exists m)(i(n, m)=0)\}, B=\{n:(\exists m)(j(n, m)=0)\}$. Let $I, J$ represent $h, i$. Let $K$ be such that $\operatorname{PRE} \vdash(K(z, y, 0)=x \&$ $K(x, y, S(z))=y)$. Let PRE $\vdash(P(0)=0 \& P(S(x))=x)$.

In view of the first three parts of Theorem 1 , it is appropriate to let SA (successor axioms) be $\{S(0) \neq 0, y \neq 0 \rightarrow(\exists x)(S(x)=y)\}$.

Lemma 11. $\mathrm{PRE}+\mathrm{SA} \vdash(K(x, y, 0)=x \&(z \neq 0 \rightarrow K(x, y, z)=y))$. $\mathrm{PRE}+\mathrm{SA} \vdash(x \neq 0 \rightarrow S(P(x))=x)$.

Proof. Arguing in PRE $+\mathrm{SA}$, if $z \neq 0$, let $z=S(a)$. Then $K(x, y, z)=K(x, y, S(a))=y$. Second part is similar.

Let $\operatorname{PRE} \vdash(L(x, y, 0)=S(y) \& L(x, y, S(z))=K(0, P(L(x, y, z))$, $I(x, L(x, y, z))))$. Let $P^{0}(x)$ be $x, P^{n+1}(x)$ be $P\left(P^{n}(x)\right)$. Let $S^{0}(x)$ be $x, S^{n+1}(x)$ be $S\left(S^{n}(x)\right)$.

LEMMA 12. PRE + SA proves the following.

(i ) $L(x, y, 0)=S(y)$.

(ii) $I(x, L(x, y, z))=0 \rightarrow L(x, y, S(z))=0$.

(iii) $I(x, L(x, y, z)) \neq 0 \rightarrow L(x, y, S(z))=P(L(x, y, z))$. 
Lemma 13. Let $0<n$. Then $\mathrm{PRE}+\mathrm{SA} \vdash(L(x, y, z)=S(0) \quad \&$ $\left.\mathbb{M}_{k<n} y \neq \bar{k}\right) \rightarrow\left(L\left(x, y, P^{n}(y)\right)=\overline{n+1} \& I(x, \overline{n+1}) \neq 0\right)$.

Proof. By induction $n$. For the basis case $n=1$, let

$$
L(x, y, z)=S(0), \quad y \neq 0 .
$$

Then $y=S(P(y))$. Hence $I(x, L(x, y, P(y))) \neq 0$. Hence $(L(x, y, y)=$ $P(L(x, y, P(y)))=S(0)$. Therefore $L(x, y, P(y))=S(S(0))$. Hence $I(x, S(S(0))) \neq 0$.

Suppose this is true for $n$. Assume $L(x, y, y)=S(0), \mathbb{M}_{k \leqq n} y \neq \bar{k}$. Then $L\left(x, y, P^{n}(y)\right)=\overline{n+1}$. Now $P^{n}(y) \neq 0$, and so $P^{n}(y)=S\left(P^{n+1}(y)\right)$. Now $\left(L\left(x, y, P^{n}(y)\right) \neq 0\right.$, and so $I\left(x, L\left(x, y, P^{n+1}(y)\right)\right) \neq 0$. Hence

$$
L\left(x, y, P^{n}(y)\right)=P\left(L\left(x, y, P^{n+1}(y)\right)\right)=\overline{n+1} .
$$

Therefore $L\left(x, y, P^{n+1}(y)\right)=\overline{n+2}$. Hence $I(x, \overline{n+2}) \neq 0$.

Lemma 14. If $n \notin A$ then $\mathrm{PRE}+\mathrm{SA} \vdash L(\bar{n}, \bar{m}, \bar{m})=S(0)$.

Proof. If $n \notin A$ then $\mathrm{PRE}+\mathrm{SA} \vdash I(n, m) \neq 0$.

Now fix $T$ to be any consistent extension of PRE + SA. Let $C=\{n: T \vdash(J(\bar{n}, y)=0 \rightarrow L(\bar{n}, y, y) \neq S(0))$.

Lemma 15. $A \subset C, C \cap B \neq \varnothing$.

Proof. Let $n \in A$. Let $i(n, m)=0$. Then $n \notin B, 1<m$. Arguing in $T$, assume $J(\bar{n}, y)=0, L(\bar{n}, y, y)=S(0)$. Now, since $n \notin B$, we see that $\mathbb{M}_{k<m-1} y \neq \bar{k}$. Hence by Lemma $13, I(\bar{n}, \bar{m}) \neq 0$, which is a contradiction. This establishes that $n \in C$. Hence $A \subset C$.

Let $n \in B, C$. Let $j(n, m)=0$. Arguing in $T$, we have $J(\bar{n}, \bar{m})=0$, $J(\bar{n}, y)=0 \rightarrow L(\bar{n}, y, y) \neq S(0)$. Hence $L(\bar{n}, \bar{m}, \bar{m}) \neq S(0)$. Since $n \in A$, we have $L(\bar{n}, \bar{m}, \bar{m})=S(0)$, by Lemma 14 . This is a contradiction. Hence $B \cap C=\varnothing$.

LEMma 16. $C$ is of the form $\{n: T \vdash M(\bar{n}, y)=0\}$, for some p.r. function symbol $M$.

Proof. Note that $\mathrm{PRE}+\mathrm{SA} \vdash(y=S(0) \leftrightarrow(y \neq 0 \& P(y)=0))$. Let $\mathrm{PRE}+\mathrm{SA} \vdash Q(x, y)=K(K(y, 0, P(y)), 0, x)$. Then PRE + SA $\vdash$ $(x \neq 0 \vee y=0 \vee P(y) \neq 0) \leftrightarrow Q(x, y)=0$. Hence PRE $+\mathrm{SA} \vdash(Q(y, x)=$ $0 \leftrightarrow(x=0 \rightarrow y \neq S(0)))$. Finally, let PRE $+\mathrm{SA} \vdash R(x, y)=Q(J(y, x)$, $L(x, y, y))$. Then $C=\{n: T \vdash R(\bar{n}, y)=0\}$.

THEOREM 4. PRE + SA is completely equationally essentially undecidable. 
Proof. Clear from Lemmas 15, 16.

CorollaRY 3. PRE $+(y \neq 0 \rightarrow(\exists x)(S(x)=y))$ is completely equationally essentially undecidable. There is a finite list of p.r. function symbols such that $\mathrm{PRE}_{X}+(y \neq 0 \rightarrow(\exists x)(S(x)=y))$ is completely equationally essentially undecidable, for all closed $X$ containing that list.

Proof. If $T$ is any theory extending

$$
\mathrm{PRE}+(y \neq 0 \longrightarrow(\exists x)(S(x)=y))
$$

not implying $x=y$, then $T$ has the same equational consequences as $T+S(0) \neq 0$. To see this, note that the models of $T$ and $T+$ $S(0) \neq 0$ are precisely the same, except that $T$ may have a one element model. For the second part, an analysis reveals that only finitely many symbols are needed for the proofs.

We now consider an extension of PRE obtained by adding the definition by cases function $\psi(a, b, c, d)=\alpha$ if $c=d ; b$ if $c \neq d$. More formally, let the extended primitive recursion indices be given by the same clauses as the primitive recursion indices, together with the clause (vi) 6 is a 4 -ary extended primitive recursion index. The extended primitive recursive function symbols (e.p.r. function symbols) are writton $F_{x}$, where $x$ is an extended primitive recursion index.

A set $X$ of e.p.r. function symbols is called closed if (i) $S \in X$. (ii) if $F_{\left(3, x, y_{1}, \cdots, y_{k}\right)} \in X$ then $F_{x}, F_{y_{1}}, \cdots, F_{y_{k}} \in X$. (iii) if $F_{(4, x, y)} \in X$ then $F_{y} \in X$. (iv) if $F_{(5, x, y)} \in X$ then $F_{x}, F_{y} \in X$. Let $\mathrm{PR}^{\prime}$ be the set of all e.p.r. function symbols.

We use $\psi$ for $F_{6}$. We let $\mathrm{PRE}^{\prime}$ be the theory whose axioms are given by the same clauses as those for PRE, together with the clauses $\psi(x, y, z, z)=x, z \neq w \rightarrow \psi(x, y, z, w)=y$.

Theorem 5. $\mathrm{PRE}^{\prime}+S(0) \neq 0 \vdash S(x) \neq x . \quad \mathrm{PRE}^{\prime}+S(0) \neq 0$ does not prove $x \neq 0 \rightarrow(\exists y)(S(y)=x)$. For closed sets $X$ containing $\psi$, every quantifier free $X$-formula is provably equivalent $\mathrm{PRE}_{X}^{\prime}$, to some equality between $X$-terms.

Proof. Assume $\mathrm{PRE}^{\prime}+S(0) \neq 0$. Let $E$ be such that $E(0)=0$, $E(S(x))=\psi(S(0), 0, E(x), 0)$. Then $E(S(x)) \neq E(x)$. Hence $S(x) \neq x$.

A model of $\mathrm{PRE}^{\prime}+S(0) \neq 0+(\exists x)(x \neq 0 \quad \& \quad(\forall y)(S(y) \neq x))$ can be constructed with domain $\{0,1,2, \cdots, \infty+0, \infty+1, \infty+2, \cdots\}$ as follows. Define 0 as $0, S(k)$ as $k+1, S(\infty+k)$ as $\infty+(k+1)$, $F_{0}$ and $F_{(2, n, m)}$ as usual, $F_{\left(3, x, y_{1}, \cdots y_{k}\right)}$ in terms of $F_{x}, F_{y_{1}}, \cdots, F_{y_{k}}$ as usual, $F_{(4, x, y)}(0)=F_{(4, x, y)}(\infty+0)=\bar{x}, F_{(4, x, y)}(n+1)=F_{(4, x, y)}(\infty+$ 
$(n+1))=F_{y}\left(n, F_{(4, x, y)}(n)\right)$, and $F_{(5, x, y)}$ analogously.

The last part is left to the reader.

We now wish to prove that $\mathrm{PRE}^{+}$is completely equationally essentially undecidable. The proof is similar to the proofs for PRE + SA, but more complicated.

LEMMA 17. There is an e.p.r. function symbol $U$ such that the following are provable in $\mathrm{PRE}^{\prime}+S(0) \neq 0$.

(i) $U(x, y, 0)=S(y)$.

(ii) $((S(P(z))=z \vee z=0) \& I(x, U(x, y, z)) \neq 0 \& S(P(U(x, y, z)))=$ $U(x, y, z)) \rightarrow U(x, y, S(z))=P(U(x, y, z))$.

(iii) $\quad((S(P(z)) \neq z \& z \neq 0) \vee I(x, U(x, y, z))=0 \vee S(P(U(x, y, z))) \neq$ $U(x, y, z)) \rightarrow U(x, y, S(z))=0$.

Proof. Left to the reader. Obviously $\psi$ is vital here. The old $K$ is not enough.

Lemma 18. $P R E^{\prime}+S(0) \neq 0 \vdash\left(\left(U(x, S(y), S(y))=S(0) \& \mathbb{M}_{k \leqq n} y \neq \bar{k}\right) \rightarrow\right.$ $\left(S\left(P^{n+1}(y)\right)=P^{n}(y) \& I(x, \overline{n+2}) \neq 0 \& U\left(x, S(y), P^{n}(y)\right)=\overline{n+2}\right)$, for $0 \leqq n$.

Proof. By induction on $n$. For the basis case $n=0$, let $U(x, S(y), S(y))=S(0), \quad y \neq 0 . \quad$ Then $U(x, S(y), S(y)) \neq 0, \quad$ and so $S(P(y))=y \vee y=0, \quad I((x, U(x, S(y), y)) \neq 0, \quad S(P(U(x, S(y), y)))=$ $U(x, S(y), y), \quad U(x, S(y), S(y))=S(0)=P(U(x, S(y), y))$. Hence $U(x$, $S(y), y)=S(S(0))$. Therefore $I(x, S(S(0))) \neq 0, S(P(y))=y$.

Suppose this is true for all $m \leqq n$. Let $U(x, S(y), S(y))=S(0)$, $\mathbb{M}_{k \leqq n+1} y \neq k$. Then $U\left(x, S(y), P^{n}(y)\right)=\overline{n+2}, S\left(P^{n+1}(y)\right)=P^{n}(y)$. Hence $U\left(x, S(y), P^{n}(y)\right) \neq 0$, and so $S\left(P^{n+2}(y)\right)=P^{n+1}(y) \vee P^{n+1}(y)=0$, $I\left(x, U\left(x, S(y), P^{n+1}(y)\right) \neq 0, S\left(P\left(U\left(x, S(y), P^{n+1}(y)\right)\right)\right)=U\left(x, S(y), P^{n+1}(y)\right)\right.$,

$$
U\left(x, S(y), P^{n}(y)\right)=P\left(U\left(x, S(y), P^{n+1}(y)\right)\right)=\overline{n+2} .
$$

Hence $U\left(x, S(y), P^{n+1}(y)\right)=\overline{n+3}$. Therefore $I(x, \overline{n+3}) \neq 0$. Now by induction hypotheses, it is easily seen that $S\left(P^{m+1}(y)\right)=P^{m}(y)$, for all $m \leqq n$. Hence $P^{n+1}(y)=0 \rightarrow y=\overline{n+1}$. Since $y \neq \overline{n+1}$, we have $P^{n+1}(y) \neq 0$, and so $S\left(P^{n+2}(y)\right)=p^{n+1}(y)$.

Lemma 19. If $n \notin A$ then $\mathrm{PRE}^{\prime}+S(0) \neq 0 \vdash U(\bar{n}, \bar{m}, \bar{m})=S(0)$.

Proof. Like Lemma 13.

Now fix $Y$ to be any consistent extension of $\mathrm{PRE}^{+}+S(0) \neq 0$. Let $C=\{n: Y \vdash(J(\bar{n}, y)=0 \rightarrow U(\bar{n}, S(y), S(y)) \neq S(0))\}$. 
Lemma 20. $A \subset Y, Y \cap B=\varnothing$.

Proof. Like Lemma 15.

THEOREM 6. $\mathrm{PRE}^{\prime}+S(0) \neq 0$ is completely equationally essentially undecidable.

Proof. Like Theorem 4.

COROLlaRY 4. PRE' is completely equationally essentially undecidable. There is a finite list of e.p.r. function symbols such that $\mathrm{PRE}_{x}^{\prime}$ is completely equationally essentially undecidable, for all closed sets $X$ of e.p.r. function symbols containing that list.

Proof. Like Corollary 3.

We now consider some forms of induction. We follow the terminology of [5] by referring to quantifier free formulae as open formulae.

Firstly, we have the axiom of induction for open formulae:

$$
(A(0) \&(\forall x)(A(x) \longrightarrow A(S(x)))) \longrightarrow A x
$$

for open $\mathrm{PR}\left(\mathrm{PR}^{\prime}\right)$-formulae $A$, which we call $\mathrm{AIO}\left(\mathrm{AIO}^{\prime}\right)$.

Secondly, we will consider the axiom of induction for equations:

$$
(\alpha(0)=\beta(0) \&(\forall x)(\alpha(x)=\beta(x) \rightarrow \alpha(S(x))=\beta(S(x))) \rightarrow \alpha(x)=\beta(x)
$$

for $\mathrm{PR}\left(\mathrm{PR}^{\prime}\right)$-terms $\alpha, \beta$, which we will call $\mathrm{AIE}\left(\mathrm{AIE}^{\prime}\right)$.

Thirdly, we have the rule of induction for open formulae:

$$
\text { from } A(0), A(x) \longrightarrow A(S(x)) \text { infer } A(x)
$$

for open $\mathrm{PR}\left(\mathrm{PR}^{\prime}\right)$-formulae $A$, which we will call RIO(RIO').

Lastly, we will consider the rule of induction for equations:

from $\alpha(0)=\beta(0), \alpha(x)=\beta(x) \longrightarrow \alpha(S(x))=\beta(S(x))$ infer $\alpha(x)=\beta(x)$

for $\mathrm{PR}\left(\mathrm{PR}^{\prime}\right)$-terms $\alpha, \beta$, which we will call $\mathrm{RIE}\left(\mathrm{RIE}^{\prime}\right)$.

By primitive recursive arithmetic (PRA), Skolem arithmetic, or recursive arithmetic, one usually means the system based on the successor axiom $S(x) \neq 0$, the equations PRE, and the rule RIO.

In PRA, the axioms PRE and $S(x) \neq 0$ are to be closed under the rule of induction. Thus we write PRA $=\mathrm{RIO}(\mathrm{PRE}+S(x) \neq 0)$. This is to be distinguished from, say, RIO(PRE) $+S(x) \neq 0$, in which PRE alone is first closed under the rule of induction, and then $S(x) \neq 0$ is added to the closure . 
LEMma 21. There are p.r. function symbols, $P, E,[x / 2],+,^{\prime},{ }^{-1}$, -, and $\oplus$ such that the following are provable in PRE + SA.

( i ) $x \neq 0 \rightarrow P(S(x))=x, P(0)=0$.

( ii ) $E(0)=0, E(x)=0 \rightarrow E(S(x))=S(0), E(x)=S(0) \rightarrow E(S(x))=0$.

(iii ) $[0 / 2]=0, E(x)=0 \rightarrow[S(x) / 2]=x, E(x)=S(0) \rightarrow[S(x) / 2]=S(x)$.

(iv ) $x+0=x, x+S(y)=S(x+y)$.

( v ) $E(x)=0 \rightarrow x^{\prime}=S(S(x)), E(x)=S(0) \rightarrow x^{\prime}=P(P(x))$.

(vi ) $E(x)=S(0) \rightarrow x^{-1}=S(S(x)),(E(x)=0 \& x \neq 0) \rightarrow x^{-1}=P(P(x))$, $0^{-1}=S(0)$.

(vii) $\quad x-0=x+x, x-S(y)=(x-y)^{-1}$.

(viii) $x \oplus 0=x, x \oplus S(y)=(x \oplus y)^{\prime}$.

LEMMA 22. The following are provable in $\mathrm{RIE}(\mathrm{PRE}+\mathrm{SA})$. (i) $x+$ $y=y+x$. (ii) $(x+y)+z=x+(y+z)$. (iii) $E(x+x)=0$. (iv) $[x+x / 2]=x$. (v) $(x+x=y+y) \rightarrow x=y$. (vi) $0 \oplus x=x+x$. (vii) $\left(x^{\prime}\right)^{-1}=\left(x^{-1}\right)^{\prime}=x . \quad$ (viii) $S(y)-x=(y-x)^{\prime} . \quad$ (ix) $x-x=0 . \quad$ (x) $x^{-1} \oplus y=(x \oplus y)^{-1}$. (xi) $(x-y) \oplus y=x+x$. (xii) $x=y \leftrightarrow x-y=0$.

Proof. (i ) $0+0=0 . \quad 0+y=y \rightarrow 0+S(y)=S(0+y)=S(y)$. Hence $0+y=y+0 . \quad S(x)+0=S(x+0) . \quad S(x)+y=S(x+y) \rightarrow$ $S(x)+S(y)=S(S(x)+y)=S(S(x+y))=S(x+S(y))$. Hence $S(x)+$ $y=x+S(y) . \quad x+y=y+x \rightarrow S(x)+y=x+S(y)=S(x+y)=$ $S(y+x)=y+S(x)$. Hence $x+y=y+x$.

(ii ) $(x+y)+0=x+y=x+(y+0) . \quad(x+y)+z=x+(y+z) \rightarrow$ $(x+y)+S(z)=S((x+y)+z)=S(x+(y+z))=x+S(y+z)=x+(y+S(z))$. Hence $(x+y)+z=x+(y+z)$.

(iii ) $\quad E(0+0)=0 . \quad S(x)+S(x)=S(S(x+x)) . \quad E(x+x)=0 \rightarrow$ $E(S(x+x))=S(0) \rightarrow E(S(S(x+x)))=0=E(S(x)+S(x))$. Hence $E(x+x)=0$.

(iv ) $[0+0 / 2]=[0 / 2]=0 . \quad E(x+x)=0, \quad E(S(x+x))=S(0)$. $E(x+x / 2)=x \rightarrow E(S(x+x) / 2)=x \rightarrow E(S(S(x+x)) / 2)=S(x)=E(S(x)+$ $S(x) / 2)$. Hence $[x+x / 2]=x$.

( v ) $x+x=y+y \rightarrow[x+x / 2]=[y+y / 2] \rightarrow x=y$.

( vi ) $\quad 0 \oplus 0=0+0 . \quad 0 \oplus x=x+x \rightarrow 0 \oplus S(x)=(0 \oplus x)^{\prime}=(x+x)^{\prime}=$ $S(S(x+x))=S(x)+S(x)$. Hence $0 \oplus x=x+x$.

(vii) By inspection.

(viii) $S(y)-0=S(y)+S(y)=(y+y)^{\prime}=(y-0)^{\prime} . \quad S(y)-x=$ $(y-x)^{\prime} \rightarrow S(y)-S(x)=(S(y)-x)^{-1}=\left((y-x)^{\prime}\right)^{-1}=y-x=\left((y-x)^{-1}\right)^{\prime}=$ $(y-S(x))^{\prime}$. Hence $S(y)-x=(y-x)^{\prime}$.

( ix ) $\quad 0-0=0 . \quad x-x=0 \rightarrow S(x)-S(x)=(S(x)-x)^{-1}=\left((x-x)^{-1}\right)^{\prime}=$ $x-x=0$. Hence $x-x=0$.

( x ) $\quad x^{-1} \oplus 0=x^{-1}=(x \oplus 0)^{-1} . \quad x^{-1} \oplus y=(x \oplus y)^{-1} \rightarrow x^{-1} \oplus S(y)=$ $\left(x^{-1} \oplus y\right)^{\prime}=\left((x \oplus y)^{-1}\right)^{\prime}=x \oplus y=\left((x \oplus y)^{\prime}\right)^{-1}=(x \oplus S(y))^{-1}$. Hence $x^{-1} \oplus y=(x \oplus y)^{-1}$. 
( xi ) $\quad(x-0) \oplus 0=(x+x) \oplus 0=x+x . \quad(x-y) \oplus y=x+x \rightarrow$ $(x-S(y)) \oplus S(y)=(x-y)^{-1} \oplus S(y)=((x-y) \oplus S(y))^{-1}=\left(((x-y) \oplus y)^{\prime}\right)^{-1}=$ $(x-y) \oplus y=x+x$. Hence $(x-y) \oplus y=x+x$.

(xii) $x=y \rightarrow x-y=0$ by (ix). $x-y=0 \rightarrow(x-y) \oplus y=0 \oplus y=$ $y+y=x+x \rightarrow x=y$ by $(\mathrm{v})$.

LEMMA 23. Every open PR-formula is provably equivalent, in $\mathrm{RIE}(\mathrm{PRE}+\mathrm{SA})$, to an equation between PR-terms.

Proof. From (xii) of Lemma 22, using the $K$ of Lemma 11.

THEOREM 7. PRA is provably equivalent to each of the following. (i) (the PR-consequences of) $\mathrm{PRE}^{\prime}+S(x) \neq 0+A I O^{\prime}$ (ii) $\mathrm{RIE}^{\prime}\left(\mathrm{PRE}^{\prime}\right)+$ $S(0) \neq 0$. (iii) $\mathrm{PRE}+S(0) \neq 0+\mathrm{AIE}$. (iv) $\mathrm{RIE}(\mathrm{PRE}+(x=0 \mathrm{~V}$ $S(P(x))=x))+S(0) \neq 0$. (v) $\operatorname{RIE}(\mathrm{PRE}+(x \neq 0 \rightarrow(\exists y)(S(y)=x)))+S(0) \neq 0$. (vi) $\mathrm{RIO}(\mathrm{PRE})+S(0) \neq 0$.

Proof. (i) $\rightarrow$ (ii), (i) $\rightarrow$ (iii) are obvious. Since $\mathrm{RIE}^{\prime}\left(\mathrm{PRE}^{\prime}\right) \vdash(x \neq$ $0 \rightarrow(\exists y)(S(y)=x))$, we have (ii) $\rightarrow$ (iv). To see that (iii) $\rightarrow$ (iv), it suffices to show that $\mathrm{PRE}+S(0) \neq 0+\mathrm{AIE} \vdash(x \neq 0 \vee S(P(x))=x)$. Arguing in PRE $+S(0) \neq 0+\mathrm{AIE}$, assume $x \neq 0, S(P(x)) \neq x$. Now $x \neq 0, x \neq y \rightarrow x \neq S(y)$. Hence $x \neq y$, which is a contradiction.

Obviously, (iv) $\rightarrow(\mathrm{v})$. Towards showing (v) $\rightarrow$ (vi), we first show that $(v) \rightarrow R I E(P R E+S A)$. This follows immediately from the fact that the set of equational consequences of $T$ is always the same as the set of equational consequences of $T+S(0) \neq 0$, whenever $T \vdash S(0)=$ $0 \rightarrow x=0$.

Next we show that RIE(PRE $+\mathrm{SA}) \vdash \mathrm{RIO}(\mathrm{PRE})$. It suffices to show that the set of consequences of RIE(PRE + SA) is closed under RIO. Assume RIE(PRE + SA) $\vdash A(0) \&(A(x) \rightarrow A(S(x)))$, where $A$ is open. By Lemma 23, let $\alpha, \beta$, be such that RIE(PRE $+\mathrm{SA}) \vdash A(x) \leftrightarrow$ $\alpha(x)=\beta(x)$. Then $\operatorname{RIE}(\mathrm{PRE}+\mathrm{SA}) \vdash \alpha(0)=\beta(0) \quad \& \quad(\alpha(x)=\beta(x) \rightarrow$ $\alpha(S(x))=\beta(S(x)))$. Hence $\mathrm{RIE}(\mathrm{PRE}+\mathrm{SA}) \vdash \alpha(x)=\beta(x)$, and so $\mathrm{RIE}(\mathrm{PRE}+\mathrm{SA}) \vdash A$. Therefore $(\mathrm{v}) \rightarrow(\mathrm{vi})$.

To see that (vi) $\rightarrow$ PRA, it suffices to prove that the set of consequences of RIO(PRE) $+S(0) \neq 0$ is closed under RIO. This is an immediate consequence of the fact that whenever an open sentence is added to a theory closed under RIO, the result is closed under RIO.

We have now shown that (i) $\rightarrow$ (ii) $\rightarrow$ (iv) $\rightarrow$ (v) $\rightarrow$ (vi) $\rightarrow$ PRA, and (i) $\rightarrow$ (iii) $\rightarrow$ (iv) $\rightarrow$ (v) $\rightarrow$ (vi) $\rightarrow$ PRA. To complete the proof, we have only to show that PRA $\rightarrow$ (i).

In [5], p. 349, it is shown that PRA $\vdash$ AIO. (We call the very interesting related Theorem 2.2 of [5] to the attention of the reader.) 
It therefore suffices to show that $\mathrm{PRE}^{\prime}+S(x) \neq 0+\mathrm{AIO}^{\prime}$ and $\mathrm{PRE}+S(x) \neq 0+\mathrm{AIO}$ have the same provable PR-formulae. It is well known that there is a p.r. function symbol $H$ which plays the role of $\psi$ in PRA; i.e., PRA $\vdash H(x, y, z, z)=x \&(z \neq w \rightarrow H(x, y, z, w)=y)$. Using $H$, we can translate each $\mathrm{PR}^{\prime}$-formula $A$ into a PR-formula $A^{*}$, (so that $A^{*}=A$. for PR-formulae $A$ ), and verify by induction that $\mathrm{PRE}^{\prime}+S(x) \neq 0+\mathrm{AIO}^{\prime} \vdash A$ implies $\mathrm{PRE}+S(x) \neq 0+\mathrm{AIO} \vdash A^{*}$.

As would be expected, PRA is not equivalent to $\mathrm{PRE}^{\prime}+\mathrm{SA}$. In fact, $\mathrm{PRE}$ ' $\mathrm{SA}$ does not prove $G(x)=S(x)$, where $G$ is introduced by $G(0)=S(0), G(S(x))=S(G(x))$. This will be an immediate consequence of Theorem 8.

A model $\mathscr{A}$ of $\mathrm{PRE}_{x}^{\prime}+\mathrm{SA}$ is called weakly saturated just in case its relational type is $X$, and for each $k+2$-ary function symbol $H \in X, 0 \leqq k$, and each $x_{1}, \cdots, x_{k}, y \in|\mathscr{A}|$, there is a sequence $\left\{y_{0}, y_{1}, \cdots\right\}$ of elements of $|\mathscr{A}|$ such that for $n \geqq 0$, we have $\mathscr{A} \vDash$ $H\left(x_{1} \cdots, x_{k}, P^{n+1}(y), y_{n+1}\right)=y_{n}$.

LEMmA 24. Every model of $\mathrm{PRE}_{x}^{\prime}+\mathrm{SA}$ has an elementary extension which is a weakly saturated model of $\mathrm{PRE}_{x}^{\prime}+\mathrm{SA}$.

Proof. By a routine use of the compactness and elementary chain theorems.

Lemma 25. Let $X$ be closed (with or without $\psi$ ). Then every model of $\mathrm{PRE}_{X}^{\prime}+\mathrm{SA}$ has an elementary extension which is a model of $\mathrm{PRE}^{\prime}+\mathrm{SA}$.

Proof. By an elementary chain argument. Let $\mathscr{A}_{0}$ be the original model of $\mathrm{PRE}_{x}^{\prime}+\mathrm{SA}$. Take $\mathscr{A}_{2 n+1}$ to be a weakly saturated elementary extension of $\mathscr{A}_{2 n}$ in the language of $\mathscr{A}_{2 n}$. Take $\mathscr{A}_{2 n+2}$ to be an expansion of $\mathscr{A}_{2 n+1}$ which satisfies $\mathrm{PRE}_{Y}^{\prime}$, where $Y$ includes all the function symbols interpreted by $\mathscr{A}_{2 n+1}$, together with all the function symbols immediately derivative of these.

THEOREM 8. Let $X$ be a closed set of extended p.r. function symbols (with or without $\psi$ ). Then $\mathrm{PRE}^{\prime}+\mathrm{SA}$ is a conservative extension of $\mathrm{PRE}_{X}^{\prime}+S A$ for all $X$-formulae.

Proof. Immediate from Lemma 25.

Another easy consequence of Theorem 8 is that the commutative law of addition is not provable in $\mathrm{PRE}^{\prime}+\mathrm{SA}$.

We conclude with a couple of open questions. 
Is the set of all open consequences of PRE decidable? We do not even know whether the set of all consequences of PRE of the form $\alpha=\beta \rightarrow S(0)=0$ is decidable.

Is $\mathrm{RIE}(\mathrm{PRE})+S(0) \neq 0$ provably equivalent to PRA? We do not even know whether its open or equational conseqences are decidable, or even whether it implies $S(x) \neq x$, or $(x \neq 0 \rightarrow(\exists y)(S(y)=x))$.

\section{REFERENCES}

1. H. P. Barendregt, Some Extensional Term Models for Combinatory Logics and i-Calculi, Dissertation, June 16, 1971, Amsterdam, p. 26.

2. A. Grzegorczyk, No Existence of recursive models for theory of combinators, Warsaw, unpublished.

3. H. Rogers, Jr., Theory of Recursive Functions and Effective Computability, McGrawHill, New York, 1967, p. 94.

4. L. Sanchis, Functionals defined by recursion, Notre Dame J. Formal Logic, 8, no. 3, pp. 161-174.

5. J. C. Shepherdson, Nonstandard models for fragments of number theory, The Theory of Models, North-Holland, 1965, pp. 342-358, Amsterdam.

6. W. W. Tait, Intensional interpretations of functionals of finite type I, J. Symbolic, Logic, 32 (1967), pp. 198-212.

Received August, 8, 1974. This research was partially supported by NSF P038823.

State University of New York-Buffalo 


\section{PACIFIC JOURNAL OF MATHEMATICS}

\section{EDITORS}

RICHARD ARENS (Managing Editor)

University of California

Los Angeles, California 90024

\section{J. DugundJI}

Department of Mathematics University of Southern California Los Angeles, California 90007

D. Gilbarg and J. Milgram

Stanford University

Stanford, California 94305
University of Washington Seattle, Washington 98105

\section{ASSOCIATE EDITORS}
E. F. BECKENBACH
B. H. NeumanN
F. WolF
K. YoShIDA

\section{SUPPORTING INSTITUTIONS}

\author{
UNIVERSITY OF SOUTHERN CALIFORNIA \\ STANFORD UNIVERSITY \\ UNIVERSITY OF TOKYO \\ UNIVERSITY OF UTAH \\ WASHINGTON STATE UNIVERSITY \\ UNIVERSITY OF WASHINGTON \\ $\stackrel{*}{*} \stackrel{*}{*} \stackrel{*}{ }{ }^{*}$ AMERICAN MATHEMATICAL SOCIETY
}

The Supporting Institutions listed above contribute to the cost of publication of this Journal, but they are not owners or publishers and have no responsibility for its content or policies.

Mathematical papers intended for publication in the Pacific Journal of Mathematics should be in typed form or offset-reproduced, (not dittoed), double spaced with large margins. Underline Greek letters in red, German in green, and script in blue. The first paragraph or two must be capable of being used separately as a synopsis of the entire paper. Items of the bibliography should not be cited there unless absolutely necessary, in which case they must be identified by author and Journal, rather than by item number. Manuscripts, in triplicate, may be sent to any one of the editors. Please classify according to the scheme of Math. Reviews, Index to Vol. 39. All other communications should be addressed to the managing editor, or Elaine Barth, University of California, Los Angeles, California, 90024.

The Pacific Journal of Mathematics expects the author's institution to pay page charges, and reserves the right to delay publication for nonpayment of charges in case of financial emergency.

100 reprints are provided free for each article, only if page charges have been substantially paid. Additional copies may be obtained at cost in multiples of 50 .

The Pacific Journal of Mathematics is issued monthly as of January 1966. Regular subscription rate: $\$ 72.00$ a year (6 Vols., 12 issues). Special rate: $\$ 36.00$ a year to individual members of supporting institutions.

Subscriptions, orders for back numbers, and changes of address should be sent to Pacific Journal of Mathematics, 103 Highland Boulevard, Berkeley, California, 94708.

\section{PUBLISHED BY PACIFIC JOURNAL OF MATHEMATICS, A NON-PROFIT CORPORATION}

Printed at Kokusai Bunken Insatsusha (International Academic Printing Co., Ltd.), 270, 3-chome Totsuka-cho, Shinjuku-ku, Tokyo 160, Japan.

\section{Copyright (C) 1975 by Pacific Journal of Mathematics} Manufactured and first issued in Japan 


\section{Pacific Journal of Mathematics}

\section{Vol. 57, No. $2 \quad$ February, 1975}

Norman Larrabee Alling, On Cauchy's theorem for real algebraic curves with boundary .......

Daniel D. Anderson, A remark on the lattice of ideals of a Prüfer domain ..................

Dennis Neal Barr and Peter D. Miletta, A necessary and sufficient condition for uniqueness of

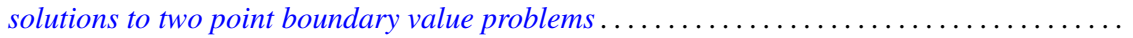

Ladislav Beran, On solvability of generalized orthomodular lattices . . . . . . . . . . ........

L. Carlitz, A three-term relation for some sums related to Dedekind sums . . . . . . . . . .....

Arthur Herbert Copeland, Jr. and Albert Oscar Shar, Images and pre-images of localization

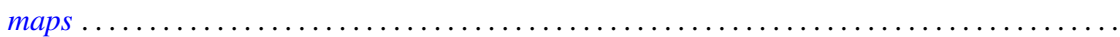

G. G. Dandapat, John L. Hunsucker and Carl Pomerance, Some new results on odd perfect

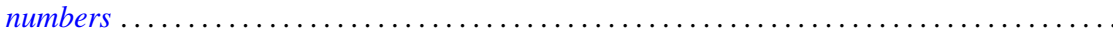

M. Edelstein and L. Keener, Characterizations of infinite-dimensional and nonreflexive

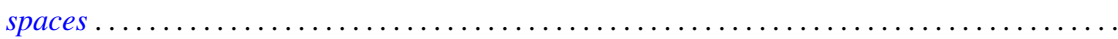

Francis James Flanigan, On Levi factors of derivation algebras and the radical embedding

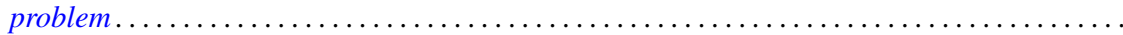

Harvey Friedman, Provable equality in primitive recursive arithmetic with and without

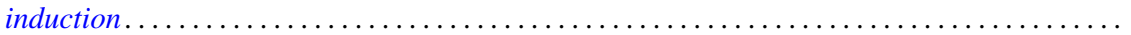

Joseph Braucher Fugate and Lee K. Mohler, The fixed point property for tree-like continua with

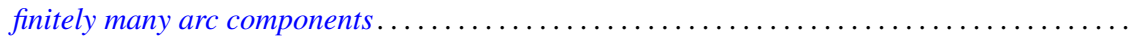

John Norman Ginsburg and Victor Harold Saks, Some applications of ultrafilters in

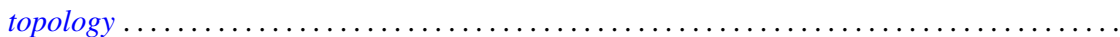

Arjun K. Gupta, Generalisation of a "square" functional equation .....................

Thomas Lee Hayden and Frank Jones Massey, Nonlinear holomorphic semigroups ..........

V. Kannan and Thekkedath Thrivikraman, Lattices of Hausdorff compactifications of a locally

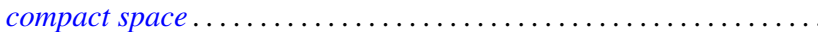

J. E. Kerlin and Wilfred Dennis Pepe, Norm decreasing homomorphisms between group

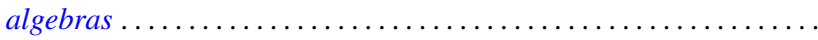

Young K. Kwon, Behavior of $\Phi$-bounded harmonic functions at the Wiener boundary ...

Richard Arthur Levaro, Projective quasi-coherent sheaves of modules .

Chung Lin, Rearranging Fourier transforms on groups...........................

David Lowell Lovelady, An asymptotic analysis of an odd order linear differential equation . . 4475

Jerry Malzan, On groups with a single involution .......................... 481

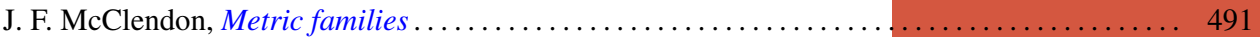

Carl Pomerance, On multiply perfect numbers with a special property .

Mohan S. Putcha and Adil Mohamed Yaqub, Polynomial constraints for finiteness of semisimple rings. .

Calvin R. Putnam, Hyponormal contractions and strong power convergence . . . . . . . . . 531

Douglas Conner Ravenel, Multiplicative operations in $\mathrm{BP} * \mathrm{BP} \ldots \ldots \ldots \ldots \ldots \ldots \ldots \ldots \ldots .539$

Judith Roitman, Attaining the spread at cardinals which are not strong limits . . . . . . . . . 545

Kazuyuki Saitô, Groups of *-automorphisms and invariant maps of von Neumann algebras . . . 553

Brian Kirkwood Schmidt, Homotopy invariance of contravariant functors acting on smooth

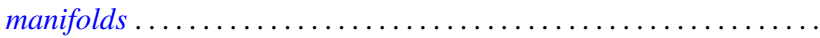

Kenneth Barry Stolarsky, The sum of the distances to $N$ points on a sphere.

Mark Lawrence Teply, Semiprime rings with the singular splitting property.

J. Pelham Thomas, Maximal connected Hausdorff spaces..............

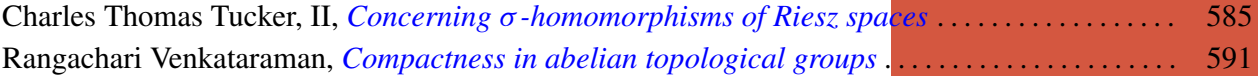

William Charles Waterhouse, Basically bounded functors and flat sheaves . . . . . . . . . . . 597

David Westreich, Bifurcation of operator equations with unbounded linearized part ......... 611

William Robin Zame, Extendibility, boundedness and sequential convergence in spaces of 